\title{
»Denn er zweifelte nicht an der Verheissung Gottes durch Unglauben« (Röm 4,20)
}

\author{
Über das »Nichtzweifeln«Abrahams
}

Benjamin Schliesser

\section{Was heisst »Zweifel«}

Unter "Zweifel« versteht man gewöhnlich »den mentalen Zustand der Ungewissheit und den Akt des Infragestellens (Bezweifelns). «" Etymologisch leitet sich das Wort "Zweifel" aus dem Zahlwort "zwei« und dem Suffix »-falt" ab, welches für die Bildung von Multiplikativzahlwörtern verwendet wird. ${ }^{2}$ In seinem Aufsatz ">An Gott zweifeln` - eine logische Untersuchung« erläutert Gunter Zimmermann:

"Die Sprachgeschichte macht klar, dass der Zweifler unsicher und ungewiss ist - Unsicherheit und Ungewissheit gehören zweifellos zu den mit `Zweifelk verbundenen Konnotationen -, weil er zwei Einstellungen zu einem [...] >Sachverhalt< besitzt. Er lehnt ihn nicht total ab, er verneint und negiert ihn nicht, er behauptet auch nicht von vornherein, dass er >nichtig $<$ oder \falsch sei, aber er akzeptiert ihn auch nicht ohne weiteres, er bejaht oder affirmiert ihn nicht, er behauptet nicht von vornherein, dass er > existent< oder >richtigく sei. Wie man in der Alltagssprache so sagt, ist er >im Zweifelk, das heisst, er steht zwischen den beiden fundamentalen Einstellungen der Bejahung oder Verneinung und hält in diesem Zustand des Zweifels beide Positionen für möglich, beide für möglicherweise >wahrı. $\aleph^{3}$

1 St. Lorenz, Art. Zweifel, Historisches Wörterbuch zur Philosophie 12 (2004), Sp. 1520-1527, 1520.

2 Vgl. G. Zimmermann, >An Gott zweifeln « - eine logische Untersuchung, NZSTh 48 (2006) 305-320, 307. Zimmermann verweist auf F. Kluge, Etymologisches Wörterbuch der deutschen Sprache (unter Mithilfe von M. Bürgisser u. B. Gregor völlig neu bearbeitet von E. Seebold), Berlin u.a. 1989, 201 (zu »-falt«, »-fältig«) und 820 (zu "Zweifel«).

3 Zimmermann, >An Gott zweifeln $<$ - eine logische Untersuchung, 307. 


\section{2. »Zweifelte« Abraham?}

Paulus kommt an zwei Stellen im Römerbrief auf den "Zweifel« zu sprechen, in Röm 4,20 und in Röm 14,23 - zumindest, wenn man gängigen Übersetzungen und Auslegungen folgt. In seinem "Abrahamkapitel « Römer 4 bietet Paulus eine "Wesensbeschreibung des Glaubens Abrahams, ${ }^{4}$ die folgenden Aspekt beinhaltet: »Denn er 'zweifelter nicht an der Verheissung Gottes durch Unglauben

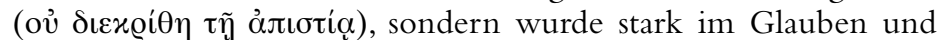
gab Gott die Ehre. $\aleph^{5}$ Folgt man der genannten Beschreibung des Zweifels, dann bringt Paulus hier zum Ausdruck, dass Abraham nicht im Zweifel war, dass er also nicht "zwischen den beiden fundamentalen Einstellungen der Bejahung oder Verneinung" stand, sondern den "Sachverhalt", d.h. den Verheissungsinhalt für möglich, für wahr hielt. In einer theologisch tiefgründigen Untersuchung über den Gedankengang des Paulus in Römer 4, äussert Joachim Jeremias:

"Wir erhalten also folgenden Sinn: [...] Abraham hat nur das verheissende Wort, nichts sonst. Aber er hält Gottes Wort für das Gewisseste, das Sicherste, was es gibt. Er klammert sich an das Wort. Er glaubt ihm, ohne sich beirren zu lassen. Mit diesem unbeirrten Vertrauen gab er Gott die Ehre. ${ }^{6}$

Diese Übersetzungs- und Auslegungspraxis wird so gut wie nie hinterfragt, obwohl es weder vorneutestamentlich noch in den ersten Jahrhunderten danach keinen (eindeutigen) Beleg dafür gibt,

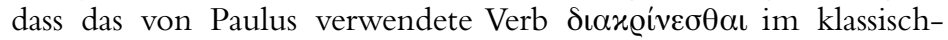
hellenistischen Gebrauch jemals die Bedeutung "zweifeln" im Sinne eines "mentalen Zustands" angenommen hat. Die gängigen

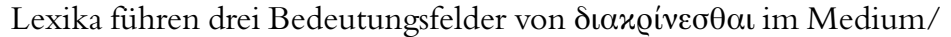
Passiv auf ${ }^{7}$ : 1. getrennt werden oder sich auflösen, 2. zu einer Ent-

\footnotetext{
4 J. Roloff, Abraham im Neuen Testament. Beobachtungen zu einem Aspekt Biblischer Theologie, in: Ders., ExegetischeVerantwortung in der Kirche.Aufsätze, hg. v. M. Karrer, Göttingen 1990, 231-254, 247.

5 So die Lutherübersetzung (1984). Die Zürcher Bibel übersetzt (2007): "An der Verheissung Gottes liess er sich durch Unglauben nicht irremachen, sondern er wurde stark im Glauben, gab Gott die Ehre."

6 J.Jeremias, Die Gedankenführung in Röm 4. Zum paulinischen Glaubensverständnis, in: Foi et Salut selon S. Paul. Épitre aux Romains 1,16, AnBib 42, Rom 1970, 51-58 (mit Diskussionsbeiträgen von E. Schweizer, M. Barth, R. Pesch, J. Cambier, St. Lyonnet u. W.C. van Unnik, 59-65), 57.

7 Vgl. die Analyse einer Reihe von Lexika und Wörterbüchern bei P. Spitaler,

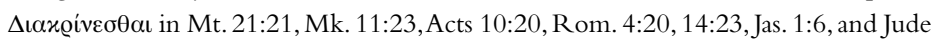


scheidung kommen und 3. sich widersetzen, widersprechen, streiten.

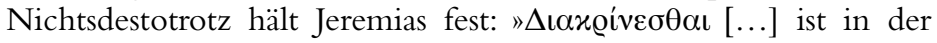
Bedeutung zzweifeln, zaudern belegt, im Neuen Testament selbst aber so stark bezeugt, dass diese Bedeutung gesichert ist. $\ll^{8}$ Er beruft sich mit seiner Aussage wie viele andere auf Walter Bauers "Griechisch-deutsches Wörterbuch", welches notiert, dass die Bedeutung "Bedenken tragen, zweifeln" "erst seit d[em] NT nachweisbar" sei. ${ }^{9}$ Es wird also davon ausge-

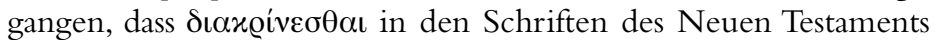
eine entscheidende Bedeutungsverschiebung erfahren hat, die zwar in einer der Grundbedeutungen des Wortes angelegt ist, aber erst in den neutestamentlichen Zusammenhängen in Erscheinung tritt: Aus der Bedeutung »streiten " - so insinuiert derWörterbucheintrag ergebe sich der innere Vorgang des "mit sich im Streite-Seins«; und daraus wiederum lasse sich die Bedeutung »Bedenken tragen" und "zweifeln« ableiten. ${ }^{10}$

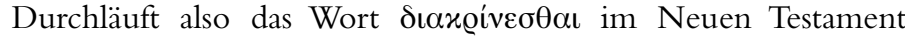
eine semantische Sonderentwicklung? Zeigt sich hier eine Eigentümlichkeit des neutestamentlichen Griechisch? Oder ist dies gar ein Beleg für die "sprachbildende Kraft des Evangeliums «" ${ }^{11}$ ? Wäre dies der Fall, so würde gewissermassen durch die Hintertür eine Variante der Philologia sacra Einzug halten, die doch spätestens seit den Bibelstudien ${ }^{12}$ Adolf Deissmanns aus dem Raum der Exegese verbannt wurde. Forschungsgeschichtlich kommt Deissmann ja das Verdienst zu, nachgewiesen zu haben, dass die Autoren des Neuen Testaments kein "Bibelgriechisch" verwendeten, sondern die weit-

22 - the 'Semantic Shift that Went Unnoticed by Patristic Authors, NT 49 (2007) 1-39, 1f. Anm. 2

8 Jeremias, Die Gedankenfuihrung in Röm 4, 56. Jeremias verweist in einer Anmer-

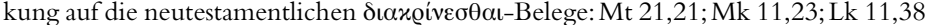
v.1. (D 05 = Codex Bezae); Apg 10,20; Röm 4,20; 14,23; Jak 1,6 (2x); Jud 22. Zu

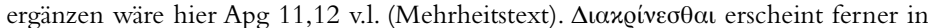
(vermeintlich) anderer Bedeutung in Apg 11,2, Jak 2,4 und Jud 9.

9 W. Bauer, Griechisch-deutsches Wörterbuch zu den Schriften des Neuen Testaments und der frühchristlichen Literatur (hg. v. K.Aland u. B.Aland), Berlin u.a. ${ }^{6} 1988$, 370 .

${ }^{10}$ Ebd.

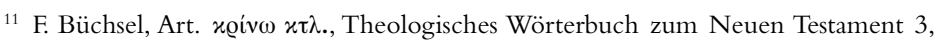
Stuttgart 1938, 920-955, 951.

${ }^{12}$ Vgl. A. Deissmann, Bibelstudien. Beiträge, zumeist aus den Papyri und Inschriften, zur Geschichte der Sprache, des Schrifttums und der Religion des hellenistischen Judentums und des Urchristentums, Marburg 1895; Ders., Neue Bibelstudien. Sprachgeschichtliche Beiträge, zumeist aus den Papyri und Inschriften, zur Erklärung des Neuen Testaments, Marburg 1897. 
hin geläufige und gebräuchliche griechische Allgemeinsprache, die sog. Koine. Ihm lag, wie er in einer "Selbstdarstellung" formulierte, an der "Säkularisation der dogmatischen Philologia sacra. ${ }^{13}$ Seine Forschungsergebnisse sollten in ein gross angelegtes Wörterbuch zum Neuen Testament münden, ${ }^{14}$ doch wurde dieser Plan erst von Walter Bauer im genannten Wörterbuch verwirklicht ${ }^{15}$ - welches

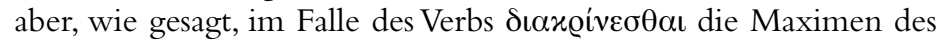
"Meisters" aus dem Blick zu verlieren schien.

So legen also schon die Epoche machenden philologischen Studien eines Adolf Deissmann eine gewisse Zurückhaltung nahe, wenn es um die Besonderheit des neutestamentlichen Griechisch und um neutestamentliche Spezialbedeutungen geht. Letztlich war es also nur eine Frage der Zeit, bis die in den Wörterbüchern und Kommentaren üblichen sprachgeschichtlichen Erklärungen in Zweifel gezogen würden. Einige wenige neuere Ansätze verzichten daher auf die Hypothese, dass das Neue Testament einen weiteren

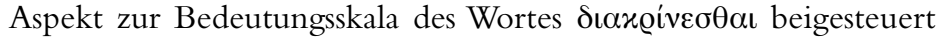
oder gar einen semantischen Sprung vollzogen hat und erklären die Belegstellen auf der Grundlage griechisch-hellenistischer Bedeutungskategorien. ${ }^{16}$ Peter Spitaler beispielsweise führt den vermeintlichen "semantic shift" nicht auf eine spezifisch neutesta-

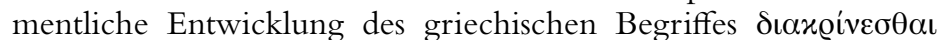
zurück, sondern hält ihn für ein Produkt des Interpretations- und Übersetzungsprozesses, der mit Origenes und Chrysostomos einsetzt. Beide Kirchenväter legen nach Spitaler eine Grundbedeutung des Wortes zugrunde, "(wider)streiten« ("contest" bzw. "dispute«), und ziehen den Zweifel interpretierend zur Erläuterung der Art und Weise des Widerstreits hinzu, ohne die beiden Vorstellungen miteinander $\mathrm{zu}$ identifizieren. ${ }^{17}$ Methodisch solle man, so schlägt Spitaler vor, vom Bedeutungsspektrum der klassisch-Hellenistischen

\footnotetext{
13 A. Deissmann, "Adolf Deissmann«, in: E. Stange (Hg.), Die Religionswissenschaft der Gegenwart in Selbstdarstellungen, Leipzig 1925, 42-78, 62.

${ }^{14}$ So Deissmann, Neue Bibelstudien, VII: »Derartige Untersuchungen auch fernerhin anzustellen, ist meine Absicht: sie sollen - sub conditione Jacobea - dereinst zu einem Wörterbuch zum Neuen Testament führen."

15 Vgl. E. Plümacher, Art. Deissmann, Adolf (1866-1937), TRE 8, Berlin u.a. 1981, 406-408, 406

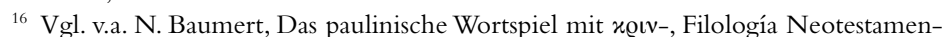
taria 15 (2002) 19-64; D. DeGraaf, Some Doubts about Doubt. The New Testament

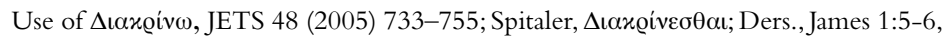
a Dispute with God, CBQ 71 (2009) 560-579. Siehe auch die ältere Arbeit von F.C. Synge, Not Doubt, but Discriminate, ExpTim 89 (1978) 203-205.

17 Spitaler, $\Delta$ เ
} 
Verwendung des Verbs ausgehen und auf Grundlage dessen die neutestamentlichen Belegstellen zu erklären suchen. Ergibt sich so ein kontextuell angemessener Sinn, sei von der These eines "semantic shift" abzusehen. ${ }^{18}$

Im Folgenden sollen nun einige Paulusinterpreten zu Wort kommen, die sich pointiert zu den relevanten Belegstellen geäussert haben und sich dabei - ihrem Sprachgefühl oder ihren philologischen Überlegungen folgend - innerhalb des traditionellen semantischen Paradigmas bewegen: Johannes Chrysostomos, Johannes Calvin und Markus Barth. Ihre Ansätze verdienen m.E. schon um ihrer selbst willen Aufmerksamkeit, doch es lässt sich zudem wahrscheinlich machen, dass ihre Auslegungen aufeinander aufbauen, die Späteren also in einem gewissen Abhängigkeitsverhältnis zu den Früheren stehen.

\section{1 »Abraham mischte sich nicht in göttliche Angelegen- heiten ein« (Johannes Chrysostomos)}

Es ist in der Tat aufschlussreich, der von Spitaler gelegten Spur zu folgen und altkirchliche Ausleger heranzuziehen, um sich deren originäre linguistische Kompetenz nutzbar zu machen. Chrysostomos' Paulusexegese galt schon bald nach seinem Tod (407 n.Chr.) als unübertrefflich; der ägyptische Mönch Isidor von Pelusium (5. Jh.) bemerkt: "Hätte der göttliche Paulus in attischer Zunge gesprochen, um sich selbst auszulegen - er hätte es nicht anders getan als dieser hochberühmte Mann. ${ }^{19}$

Chrysostomos kommt in einer Vielzahl von Zusammenhängen auf Röm 4,20 zu sprechen. Immer zeigt sich darin auch der pragmatische Charakter seiner exegetischen Erörterungen, die zwar je nach Zusammenhang unterschiedlich akzentuiert sind, doch stets darauf abzielen, vor einer anmassenden Neugierde gegenüber dem göttlichen Wesen und Handeln zu warnen. In seiner neunten Homilie zum Römerbrief ${ }^{20}$ rühmt er den Glauben Abrahams, der

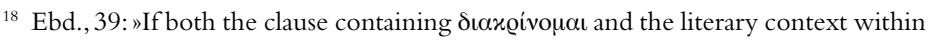
which the clause is embedded support a traditional meaning of the verb, an argument for a semantic shift is without merit; a rendering of the verb according to classical/ Hellenistic Greek conventions needs to be pursued."

${ }^{19}$ M. Mitchell, The Heavenly Trumpet. John Chrysostom and the Art of Pauline Interpretation, HUTh 40, Tübingen 2000, 31 .

${ }^{20}$ Text in PG 60, 461. Es handelt sich um die 9. bzw. 8. Homilie (zumeist wird in deutschen und englischen Übersetzungen die Einleitung als erste Homilie gezählt, so dass sie gegenüber der PG um eine Ziffer abweichen, die ihre Zählung mit der ersten Homilie beginnt). Deutsche Übersetzung in: Des heiligen Kirchenlehrers Johannes 
"wider die menschliche Hoffnung auf die Hoffnung Gottes hin" glaubte. Den Satz des Paulus, dass Abraham »an der Verheissung Gottes nicht im Unglauben rzweifelte«", legt Chrysostomos im Sinne dieser Gegensätzlichkeit zwischen menschlicher und göttlicher Betrachtungsweise aus:

"Weder einen Beweis gab Gott, noch wirkte er ein Zeichen, sondern es waren lediglich blosse Worte, die das verhiessen, was die Natur nicht versprach. Und doch heisst es: Oひ̉

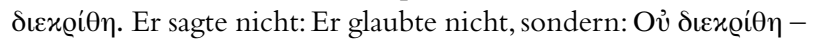
das heisst: er überlegte nicht nach zwei Seiten hin und her (oủdè

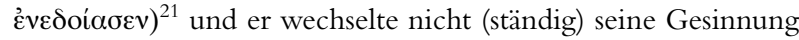
bzw. seinen Standpunkt (oủdè $\alpha \dot{\mu} \phi \dot{\varepsilon} \beta \alpha \lambda \varepsilon$ ), ${ }^{22}$ obwohl doch die Hindernisse so gross waren."

Und was tat Abraham? Er "gab Gott die Ehre und hatte die feste Gewissheit: Er vermag, was er verheissen hat, auch zu tun« (Röm 4,20-21). In seiner Erklärung dieser Passage tritt eine zentrale Anschauung des Chrysostomos zutage, die sich durch sein ganzes Werk zieht und zugleich das "Nichtzweifeln" Abrahams konturiert: Gott die Ehre geben, so legt er dar, heisst, seine Kraft nicht unnütz in Dinge zu investieren, die einen nichts angehen bzw. die das eigene Fassungsvermögen übersteigen ( steht uns schon nicht an, uns in Dinge von geringerer Bedeutung einzumischen und darüber Untersuchungen anzustellen

Chrysostomus, Erzbischofs von Konstantinopel, Kommentar zum Briefe des Hl. Paulus an die Römer, I. Teil (übers. v. J. Jatsch), BKV 39, München 1922.

${ }^{21}$ Von dotós "zweifach, doppelt, zwei».

${ }^{22}$ Im biblischen Gebrauch (LXX und NT) bedeutet $\alpha \mu \phi ı \beta \alpha \lambda \lambda \lambda \varepsilon v$ mauswerfen t.t. v. Auswerfen d. runden Wurfnetzes" (Bauer, Griechisch-deutsches Wörterbuch zu den Schriften des Neuen Testaments und der frühchristlichen Literatur, 92) (Hab 1,17; Mk 1,16; vgl. Jes 19,8). Wörterbücher verweisen häufig auf Aristoteles, Ethica Eudemia 1243a 25, wo man am besten »den Standpunkt wechseln« übersetzt (vgl. die Übersetzung bei F. Dirlmeier, Eudemische Ethik, Werke in deutscher Übersetzung/ Aristoteles 7, Berlin ${ }^{4}$ 1984, 86f.). Das Verb nimmt aber auch die Bedeutung "zweifeln"

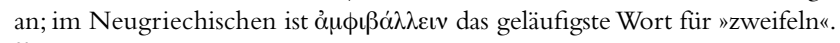

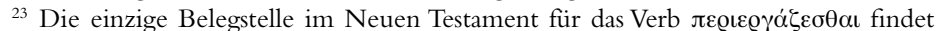
sich in 2Thess 3,11 (das Nomen noch in Apg 19,19 und 1Tim 5,13). Zur Auslegung vgl. B.W.Winter, Seek the Welfare of the City. Christians as Benefactors and Citizens, Grand Rapids 1994, 48-72, v.a. 50: "The context [...] suggests that it is much more likely to be a description of the activity of a client supporting his patron's cause in politeia. Epictetus commends the person in public life who is neither a sbusybody

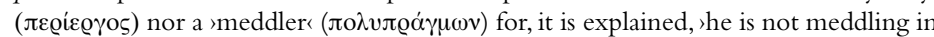
other people's affairs when he is overseeing the actions of men when this is his proper [official] concern $(\tau \alpha ̉$ ı̌dı $\alpha)<(I I I .22 .97)$." 


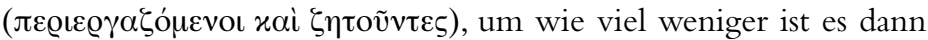
an uns, an einer solchen Sache wie der Geburt des Herrn herum-

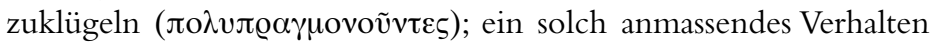

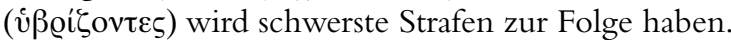

Das Motivfeld des unbotmässigen Erforschens und sündigen

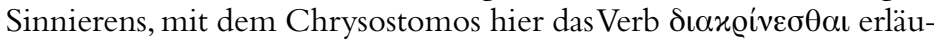
tert, lässt sich bei Chrysostomos an zahlreichen Zusammenhängen belegen, die alle davor warnen, sich in frevelhafter Weise mit dem göttlichen Werk und Wesen auseinanderzusetzen, sich also in Dinge einzumischen, die dem Menschen in seiner Begrenztheit nichts angehen. ${ }^{24}$ Derartige Äusserungen stehen im Zusammenhang seiner Auseinandersetzung mit den Gegnern des Konzils von Nizäa. Chrysostomos wurde ja in den Nachbeben dieses Konzils geboren und erlebte die Höhepunkte des arianischen Streits. Die recht unvermittelt auftauchende Reminiszenz an die Geburt Christi ist jener christologischen Debatte geschuldet. An einer anderen Stelle, nämlich in einer Reihe von Predigten »über die Unbegreiflichkeit Gottes", beruft sich Chrysostomos wieder auf Abraham als das Vorbild der Glaubenden: Dieser stellt die Verheissung Gottes nicht infrage ( $\mu \grave{\eta}$

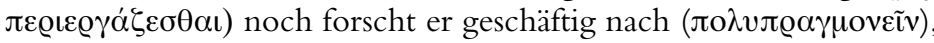
sondern er gehorcht und glaubt und gibt damit Gott die Ehre. ${ }^{25}$ Eben deshalb wurde Abraham gerechtfertigt, weil er die Neugier des Wissens ablegte und schlicht glaubte! Denn
"wenn Gott uns etwas offenbart, das nicht kritisch hinterfragt

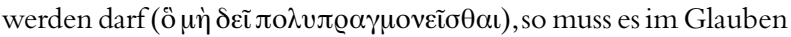

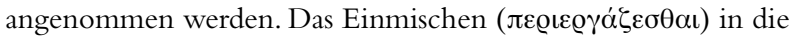

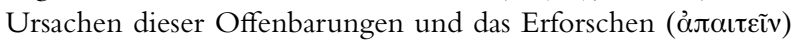

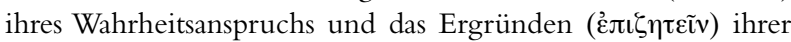 Verwirklichung - das ist das Werk einer schamlosen und rück- sichtslosen Seele. $\|^{26}$

${ }^{24}$ Vgl. Jean Chrysostome. Sur l'incompréhensibilité de Dieu. Homélies I-V, SC 28

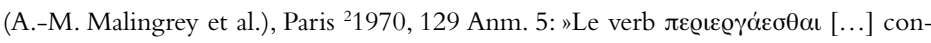

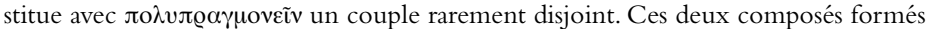
de deux verbes à sens très voisins ne diffèrent que par leurs préverbes, l'un soulignant la multiplicité des questions posées [...], l'autre des démarches multiples autour d'un sujet [...]. Mais l'un et l'autre sont toujours affectés d'un coefficient péjoratif, cette activité étant présentée comme parfaitement vaine, parce que son objet dépasse l'intelligence de l'homme. Daneben R. Lim, Public Disputation, Power, and Social Order in Late Antiquity, The Transformation of the Classical Heritage 23, Berkeley 1995, 174.

${ }^{25}$ De incomprehensibili 2, 304-326 (SC 28, 166-169). Gedankengang und Begrifflichkeit entsprechen auffällig der Homilie zu Röm 4,19-21.

${ }^{26}$ Ebd., 77-79 (SC 28, 148f.). 
Es ergibt sich also folgendes Verständnis: Abraham hat sich Gott gegenüber nicht anmassend verhalten und sich in Dinge eingemischt, die ihn nichts angehen. Er hat sich Gottes Göttlichkeit nicht wichtigtuerisch widersetzt.

\section{2 »Abraham untersuchte die göttliche Verheissung nicht misstrauisch« (Johannes Calvin)}

Johannes Calvins Schriftauslegung ist

»entscheidend beeinflusst vom Bibelhumanismus zu Beginn des 16. Jahrhunderts. Schon in Paris lernt Calvin die Ideale des Bibelhumanismus kennen und beginnt seinerseits, die Bibel in den Ursprachen auszulegen [...]. Von Erasmus übernimmt er weithingehend philologische und textkritische Einsichten. ${ }^{27}$

In seiner Baseler Zeit (ab 1535) und v.a. in Strassburg (15381541) widmete sich Calvin intensiven patristischen Studien. Die erste Auflage des Römerbriefkommentars veröffentlicht er im Jahr $1540,{ }^{28}$ also kurz nach der zweiten Fassung der "Institutio«. "Alle Schriften lassen deutlich den wachsenden Einfluss der patristischen Literatur innerhalb der calvinistischen Theologie erkennen. $\otimes^{29}$ Calvin war u.a. im Besitz einer lateinischen Gesamtausgabe der Werke des Chrysostomos, die er wohl während seiner Strassburger Zeit studier$t^{30}$ und (ganz im Stil der populären Lektüreanweisungen des Erasmus) mit Unterstreichungen und Anmerkungen versah. ${ }^{31}$ Neben Augustin war Chrysostomos derjenige Kirchenvater, der Johannes Calvins eigene Arbeiten massgeblich inspirierte. In exegetischen Belangen verliess sich Calvin jedoch (entsprechend seines Selbstzeugnisses) in weit grösserem Masse auf Chrysostomos als auf Augustin, ${ }^{32}$ und

\footnotetext{
${ }^{27}$ K.-H. zur Mühlen, Der Begriff "sensus« in der Exegese der Reformationszeit (1996), in: Ders., Reformatorische Prägungen. Studien zur Theologie Martin Luthers und zur Reformationszeit (hg. v. A. Lexutt u.V. Ortmann), Göttingen 2011, 96-110, 110.

${ }^{28}$ Überarbeitete Auflagen erschienen 1551 und 1556.

29 A. Ganoczy u. K. Müller, Calvins handschriftliche Annotationen zu Chrysostomus. Ein Beitrag zur Hermeneutik Calvins, Veröffentlichungen des Instituts für Europäische Geschichte Mainz 102,Wiesbaden 1981, 25.

${ }^{30}$ Vgl. Ganoczy u. Müller, Calvins handschriftliche Annotationen zu Chrysostomus, 27.

31 Calvin besass Claude Chevallons Ausgabe der Opera omnia aus dem Jahr 1536 (vgl. Ganoczy u. Müller, Calvins handschriftliche Annotationen zu Chrysostomus, 4-17).

${ }^{32}$ Vgl. A.N.S. Lane, John Calvin. Student of the Church Fathers, Edinburgh 1999, 39
} 
plante gar eine französische Ausgabe seiner Homilien; auch wenn es nicht zur Ausführung des Plans kam, so gibt doch die "Praefatio in Chrysostomi homilias" Auskunft über die Hochachtung Calvins vor Chrysostomos' exegetischen Fähigkeiten. ${ }^{33}$ Er sei der "vorzüglichste (Ausleger) von allen. ${ }^{34}$

Zwar findet sich in Calvins Chrysostomosausgabe lediglich eine Unterstreichung in den Homilien zum Römerbrief - also nicht $\mathrm{zu}$ Röm 4,20 -, doch hat Calvin sicher wauch andere Chrysostomossammlungen bzw. Quellen, in denen Chrysostomostexte schon verarbeitet waren, gekannt $\ll^{35}$ und konnte sich bei seinen Studien eine umfassende Kenntnis der Kirchenväter und insbesondere der Werke des Chrysostomos aneignen. Die vorzügliche Kenntnis und Hochachtung Calvins gegenüber dem Kirchenvater legen also nahe, dass dessen Einsichten auch in die Auslegung des Römerbriefes eingeflossen sind. Nicht zuletzt findet sich in den von Calvin sehr genau gelesenen und umfassend kommentierten Genesishomilien eine markierte Passage, die davor warnt, sich neugierig in die göttlichen Angelegenheiten einzumischen und zu sagen: "Warum schuf Gott dies oder jenes? « ${ }^{36}$ und eine weitere, ebenfalls angestrichene Stelle empfiehlt, nicht nach dem Ort der Entrückung Henochs zu fragen, sondern schlicht das Gesagte zu glauben. ${ }^{37}$ Somit lässt sich eine Reminiszenz des antiochenischen Kirchenvaters in Calvins Auslegung von Röm 4,20 durchaus wahrscheinlich machen.

In seinem eigenen Kommentar zum Römerbrief erklärt Calvin die Glaubensstärke Abrahams so, dass Abraham "nicht geschwankt habe und nicht hin und her wog (non vacillasse, aut fluctuatum esse)."

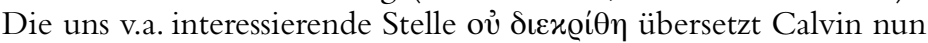
aber anders als die Vulgata mit "non disquisivit ${ }^{38}$ und tut dies, wie er in der Auslegung erläutert, nicht ohne Grund:

33 Vgl. CR 37, Sp. 831-838.

${ }^{34}$ CR 37, Sp. 834 ("Chrysostomum ex omnibus potissimum delegerim»).

${ }^{35}$ Ganoczy u. Müller, Calvins handschriftliche Annotationen zu Chrysostomus, 24.

${ }^{36}$ Vgl. Ganoczy u. Müller, Calvins handschriffliche Annotationen zu Chrysostomus,

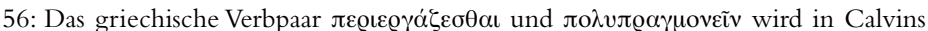
Ausgabe mit »obmurmurare, et curiosius inquirere« übersetzt.

${ }^{37}$ Vgl. Ganoczy u. Müller, Calvins handschriftliche Annotationen zu Chrysostomus,

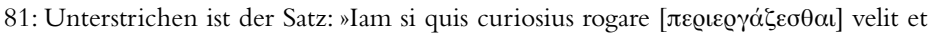
dicere: et quo ipsum [sc. Enoch] transtulit?

${ }^{38}$ Die Vulgata bietet "non haesitavit«. - Der lateinische Text (der dritten Ausgabe aus dem Jahr 1556) von Calvins Römerbriefkommentar ist abgedruckt in Ioannis Calvini Commentarius in Epistolam Pauli ad Romanos (hg. v. T.H.L. Parker u. D.C. Parker), Ioannis Calvini Opera Omnia [...] Series II: Opera Exegetica Veteris et Novi Testa- 
"Auch wenn ich den alten Interpreten und Erasmus nicht folge, entbehrt meine Version nicht der Begründung. Denn es scheint, dass der Apostel sagen wollte, Abraham habe die Waage des Unglaubens nicht abgewogen, ob Gott das gewähren könne, was er verheissen hat. Denn eigentlich untersuchen (disquirere) wir alles, wenn wir es aufgrund von Misstrauen erforschen (excutimus), und wollen nur das zulassen, was uns nach intensiver Prüfung glaubwürdig (credibile) erscheint.»

Freilich erkennt Calvin die Diskrepanz seiner Auslegung mit dem Lachen Abrahams angesichts der Verheissung (Gen 17,17), doch er fasst Abrahams Reaktion - anders als Saras (Gen 18,12-15) - als einen Ausdruck der Verwunderung: Zwar habe Abraham gefragt, "wie das wohl geschehen könne; aber das war eine Frage derVerwunderung, so wie bei der Jungfrau Maria, als sie vom Engel zu erfahren suchte, wie geschehen könne, was er angekündigt hatte, und dergleichen."Seine reformatorische Zuspitzung erfährt Calvins Exegese, wenn er erklärt,

"dass wir Gott nicht mehr Ehre geben können, als wenn wir seine Wahrheit im Glauben versiegeln [...] Deshalb ist das Allerwichtigste bei seiner Verehrung, seine Verheissungen gehorsam anzunehmen; die wahre Gottesverehrung fängt mit dem Glauben an (Veraque religio a fide incipit)."

Calvins Ausführungen zufolge hat Abraham also die Zusage Gottes nicht einer misstrauischen Prüfung unterzogen. Er ist Gott nicht mit hoffärtigen Fragen und ungehorsamen Untersuchungen entgegen getreten.

\section{3 »Abraham leistete nicht aktiven Widerstand gegen Gott» (Markus Barth)}

In dem Tagungsband, der Joachim Jeremias' bereits erwähnten Vortrag dokumentiert, findet sich eine erwähnenswerte, wohl ad hoc formulierte Replik des reformierten Neutestamentlers Markus Barth auf die knappen Ausführungen Jeremias' zum "Zweifel" Abrahams. Die Argumentation dieser Replik atmet den Geist der Calvinschen Deutung, ist aber ergänzt durch lexikographische Hinweise und die Überführung des "Settings" in einen juridischen Kontext. Barth hält zunächst fest, dass er die Darbietung Jeremias'

menti 13, Genf 1999. Die deutsche Übersetzung folgt der Calvin-Studienausgabe (hg. v. E. Busch, u.a., 5/1: Der Brief an die Römer. Ein Kommentar, Neukirchen-Vluyn, 2005, 247-253. 
für »eine klassische Darbietung der Linie von Augustin zu Luther und $\mathrm{zu}$ allen braven Bekennern, nicht nur der Reformation, sondern auch unter den Katholiken « hältt. ${ }^{39}$ Doch seine Kritik setzt da ein, wo ihm der Glaube als "Haltung der $\psi v \chi \eta_{\eta} \ll$, als ein »dennochJa-sagen " entgegentritt, dessen Gegenteil der Zweifel sei. ${ }^{40}$ Das Alte Testament kenne kein Wort für "Zweifel«, sondern bringe das Gegenteil des Glaubens - hebr. hä'ämin = „fest sein«, "sich fest machen" - durch die Vorstellungen des "Schwankens" oder "Wankens" zum Ausdruck. ${ }^{41}$

"Wenn in Röm 4 Zweifel das Gegenstück zu Glaube ist, so wäre das eine Innovation von Paulus, die recht schlecht passte zu einer Demonstration der Natur des Glaubens mit Hilfe von Abraham und Alttestamenttexten! Wenn Paulus alttestamentlich argumentiert, und wenn er ein einigermassen guter Exeget ist, dann muss er in der Linie des Alten Testaments argumentieren. Wenn er aber etwas so Neues einträgt wie den griechischen Begriff des Zweifelns gegenüber der Sicherheit des Glaubens, dann missbraucht er eigentlich das Alte Testament. ${ }^{42}$

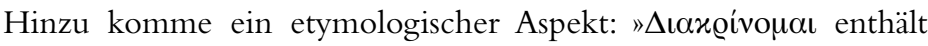
nicht die Wurzel Zwei oder Entzweiung (ein Gegensatzpaar)« - wie etwa "zweifeln«, »douter" oder »to doubt« - »sondern etymologisch bedeutet es: hier ist jemand, der ein Urteil fällt und hier ist jemand, der eine Antwort darauf gibt $<$ - und zwar eine negative Antwort, der das Urteil zurückstösst zu seinem eigenen Unheil. «33 Man komme der paulinischen Aussageabsicht am nächsten, wenn man übersetzte: Abraham

»hätte sich zum Gegenrichter gegen die Verheissung Gottes gemacht, wenn er nicht geglaubt hatte [sic], oder: er stellte sich selbst aber nicht als ein Besserwisser Gott gegenüber. Das würde dann aus der Psychologie des Zweifelns hinwegführen und würde zurückführen zu dieser Gerichtssituation [...]: Hier ist Gott mit seinem verheissenden Spruch und der Frage: Was macht der Mensch diesem Spruch gegenüber? Sagt er: Ich lasse mir diesen Spruch recht sein? ${ }^{44}$

${ }^{39}$ M. Barth, in: Jeremias, Die Gedankenführung in Röm 4, 59

${ }^{40}$ Ebd., 59.

${ }^{41}$ Ebd., $59 f$.

${ }^{42}$ Ebd., 60.

${ }^{43}$ Ebd., 60.

${ }^{44}$ Ebd., 60. 
An einer anderen Stelle der Diskussion nimmt Barth diesen Faden wieder auf und fasst seine Einwände gegen das traditionelle Verständnis zusammen:

"Das Gegenteil von Glaube ist, dass man Gottes Rechtsspruch oder Rechtsordnung nicht annimmt, nicht daraus lebt sondern, dass man es besser weiss. Also, ich würde sagen, dieses Widersprechen, sich selbst zum Gegen-Richter aufsetzen, ist etwas Anderes als dass man zweifelt und sagt; 'Soll ich es annehmen, soll ich es nicht annehmen? Es ist aktiver Widerstand gegen Gott. « ${ }^{45}$

Folgt man Barths Gedankengang, dann stellte sich Abraham nicht als ein »Besserwisser" Gott gegenüber; er hat Gott nicht widersprochen und sich nicht zum Gegenrichter aufgesetzt.

\section{3. "Zweifel« und »Widerstreit»}

Ohne Zweifel offenbaren die genannten Auslegungen ihre Kontextgebundenheit und die eigenen rhetorischen Absichten ihrer Urheber - zu denken ist an den pädagogischen und kirchenpolitischen Impetus des Chrysostomos angesichts der christologischen Kontroverse, ${ }^{46}$ an das "spezifisch ekklesiale[ Denken Calvins, das seine reformerische Intention nicht verbirgt ${ }^{47}$ und an die sich der Dialektischen Theologie verdankende Aversion Markus Barths gegen ein "psychologisches" Glaubensverständnis. Doch unabhängig von ihren jeweiligen "Vorverständnissen« und Aussageabsichten liefern ihre Ausführungen einen beachtenswerten philologisch-exegetischen Beitrag zur paulinischen Verwendung

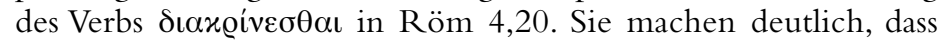
sich auf dem Hintergrund herkömmlicher Verwendungstypen des Wortes alternative Verstehensmöglichkeiten der paulinischen Aussagen eröffnen, die nicht ohne exegetische und systematischtheologische Relevanz sind. Möglicherweise lag Paulus weniger am heroischen, unbeirrbaren "Dennochglauben ${ }^{48}$ des Abraham oder an dessen "mentalem Zustand der Gewissheit" als vielmehr am Gedanken, dass Abraham nicht wie »die gefallene adamitische

\footnotetext{
${ }^{45}$ Ebd., 65.

${ }^{46}$ Vgl. zu den rhetorischen Absichten des Chrysostomos Mitchell, The Heavenly Trumpet, 30 .

47 Ganoczy u. Müller, Calvins handschriftliche Annotationen zu Chrysostomus, 22.

48 A. Deissmann, Paulus. Eine kultur- und religionsgeschichtliche Skizze, Tübingen $21925,127 f$.
} 
Menschheit« (vgl. Röm 1,18-32) mit »verfinstertem Herz« (1,21) eigene Weisheit beanspruchte $(1,22)$ und sich der göttlichen Schöpfungsabsicht und -ordnung entgegen stellte $(1,22-25)$ und dass er nicht wie "einige Juden" (vgl. 3,1-8) die "Wahrheit Gottes" verwarf $(3,3)$ und sich den "Worten Gottes" widersetzte $(3,4)$. Das Gegenbild des Glaubens wäre nach Paulus folglich weniger der "Zweifel" am Verheissungsinhalt als vielmehr der "Widerspruch" gegen den, der verheisst. ${ }^{49}$

In einem eigenen Argumentationsgang wäre nun zu entfalten, wie sich "Zweifel " und "Widerspruch" gegenüber Gott und seiner Verheissung zueinander verhalten. Ein Hinweis möge an dieser Stelle genügen: In seiner grossen Genesisvorlesung (1535-1545) erläutert Martin Luther, dass der Fall Adams mit der Frage der scheinheiligen Schlange einsetzt: "Sollte Gott gesagt haben?» (Gen 3,1). Seine Sünde bestand nicht im Essen der Frucht, sondern darin, dieser Frage Raum zu geben.

"Sünde heißt: Gottes Zusage nicht zu trauen, an ihr zu zweifeln: Hält Gott auch, was er verspricht? Sollte Gott gesagt haben? Luther nennt dies >disputare de deo . Mit Gott disputieren und dabei zweifeln kann zwar auch der angefochtene Glaube eben sofern er seine Fragen als Klage vor Gott bringt. Unglaube aber wird dieser Zweifel, wenn er sich aus dem Gottesverhältnis löst und in selbstrechtfertigender, selbststabilisierender Absicht laut wird.Wer die Erfüllung seines Lebens selbst in die Hand nehmen, sich selbst garantieren will, meint, das Hören und Antworten als Loben, Klagen und Bitten - nicht mehr nötig zu haben. $"{ }^{50}$

Adam beanspruchte Autonomie, stellte sich ausserhalb Gottes und machte so den Anspruch Gottes auf den Menschen "zum disputablen Problem $"{ }^{51}$ Es gibt also nach Luther eine Form des Zweifels, die in eine sich distanzierende "Auseinander-Setzung « übergeht; »dubitare« führt ins »disputare«. Darin greifen "Zweifel" und »Widerspruch" ineinander - und darin liegt der entscheidende Unterschied zwischen Adam, dem "paradigmatischen Sünder«, und dem "Glaubensvorbild» Abraham: Im Gegensatz zu Adam stellte sich Abraham nicht ausser-

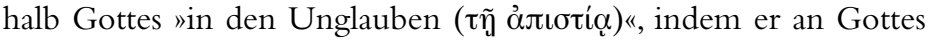

${ }^{49}$ Eine nähere exegetische Begründung dieser Gegenüberstellung Röm 4,20 und 1,18-32 bzw. 3,1-8 wird an anderer Stelle folgen.

${ }^{50}$ O. Bayer, Martin Luthers Theologie. Eine Vergegenwärtigung, Tübingen ${ }^{3} 2007$, 162.

${ }^{51}$ R. Bultmann, Welchen Sinn hat es, von Gott zu reden (1925), in: Ders., Glauben und Verstehen. Gesammelte Aufsätze, Bd. 1, Tübingen ${ }^{9} 1993,26-37,27$. 
Benjamin Schliesser

Wort zweifelte und in eine Auseinandersetzung mit ihm und über ihn trat, sondern er blieb »im Glauben", »wurde stark im Glauben und gab Gott die Ehre."

—Dr. Benjamin Schliesser ist Oberassistent an den Lehrstühlen für Neutestamentliche Wissenschaft an der Universität Zürich. 